\title{
Quantitative Response of Cucumis melo Inoculated with Root Rot Pathogens
}

\author{
M. Biernacki, Department of Horticulture and Landscape Architecture, Oklahoma State University, Lane 74555 - \\ 0128 and B. D. Bruton, United States Department of Agriculture-Agricultural Research Service, South Central Ag- \\ ricultural Research Laboratory, Lane, OK 74555
}

\begin{abstract}
Biernacki, M., and Bruton, B. D. 2001. Quantitative response of Cucumis melo inoculated with root rot pathogens. Plant Dis. 85:65-70.

This experiment quantified the effects of three root rot pathogens on muskmelon (Cucumis melo L., var. cantalupensis) growth traits using computerized image analysis. Plants were grown from seed in sand infested with the soilborne pathogen Monosporascus cannonballus, Acremonium cucurbitacearum, or Rhizopycnis vagum. After 28 days in the growth chamber, images of plants were analyzed to quantify their response. Compared to noninoculated muskmelons, inoculated plants had significantly increased mean root diameter $(45 \%)$, decreased root length ( $26 \%$, primarily in roots of $<0.5 \mathrm{~mm}$ diameter), decreased number of root tips (27\%), decreased rhizosphere volume (40\%), and decreased cumulative and mean surface area of leaves $(24 \%)$. Effects of M. cannonballus on muskmelon growth were significantly different compared to $A$. cucurbitacearum and $R$. vagum. Isolate effects manifested a greater magnitude of difference on muskmelon traits than those observed at the species level. Multivariate analyses of plant responses were more powerful than univariate analyses to differentiate among effects of pathogen species and pathogen isolates. Discriminant analysis were useful to identify groups of plant traits modified by each fungal species or isolate at low disease levels. Digital image analyses proved to be a useful technique in quantitative assessment of plant damage caused by soilborne root rot pathogens.
\end{abstract}

Additional keywords: cantaloupe, Cucurbitaceae, vine decline
Roots are not amenable to study due to their growth in nontransparent growing media and spatial properties like numerous modular roots, hierarchical branching, small diameters, and root tapping (2). In addition, factors such as light condition, experience of rater, visual illusions, and complex three-dimensional shapes may thwart root evaluation $(7,11,15)$. Keys and templates have been introduced to assist with qualitative and quantitative evaluation of plant damage to ameliorate errors associated with visual evaluation of plant disease $(7,16)$. The most common destructive measures of root disease by soilborne pathogens have historically included root mass, tap root length, volume of rhizosphere, and estimates of total root length $(7,11,15)$. Nondestructive evaluation methods of root disease are based on the assumption that damage to underground plant tissues is correlated with aboveground plant growth traits $(7,11)$. Aboveground characteristics used to evaluate plant health include aboveground biomass, variation in

Corresponding author: B. D. Bruton

Accepted for publication 30 September 2000.

Publication no. D-2000-1106-03R

This article is in the public domain and not copyrightable. It may be freely reprinted with customary crediting of the source. The American Phytopathological Society, 2001.
E-mail: bbruton-usda@lane-ag.org color, leaf number, respiration, leaf area, plant height, canopy density and volume, and timing of growth stages $(7,11)$. Howent measures (particularly of roots) to assess plant heath as well as the variability of various plant characteristics $(7,10,11)$. Difficulties with manual root measurements limit sample number and may lower probability to detect significant treatment effects (7).

Soilborne pathogens incite root injury and cause disease that affects plant growth, development, and reproductive output of cucurbits (6). Fungi attack plant roots and modify their characteristics and pattern of growth and development. Depending on the pathogen and mode of action, effects on roots and plant can be quite different (3-6). Unfortunately, our understanding of pathogen effects on root ecology is limited ever, rarely have studies compared differ- due to the complex nature of root studies in soil and due to inadequate methodology addressing these problems (17). Collection of information on root traits other than mass is often constrained by lack of simple and efficient quantitative methodology, applicable to the wide range of needs, and due to technical and financial limitations (2). Digital imaging may have potential in the study of root diseases that circumvents some previous technical limitations. Digital imaging and image analysis may aid in overcoming many problems associated with visual estimation and manual measurement of plant samples (15). The objective of the present study was to quantify plant traits most responsive to effects of fungal root rot pathogens at species and isolate levels using digital imaging.

\section{MATERIALS AND METHODS}

Fungal inoculation. Two isolates each of three species of fungi (Acremonium cucurbitacearum, Monosporascus cannonballus, and Rhizopicnis vagum), previously isolated from muskmelon roots exhibiting symptoms of vine decline, were used in the study. Using the inoculation method described by Bruton et al. (4), methyl bromide-sterilized river sand for six pots was infested with an isolate of each test fungus at inoculum densities indicated in Table 1.

Two replicate studies were performed. Each study consisted of 24 pots with plants, including 6 control pots, 6 pots inoculated with A. cucurbitacearum, 6 pots inoculated with $M$. cannonballus, and 6 pots inoculated with $R$. vagum. Each pathogen species was represented by two isolates with three pot replicates per isolate. Five seeds of Cucumis melo var. cantaloupensis (cv. Hymark) were placed on top of the sand, and an additional $4 \mathrm{~cm}$ of infested sand was added to cover seeds and fill the pot $(14.5-\mathrm{cm}$ height by $11.0-\mathrm{cm}$
Table 1. Fungal isolates of root rot pathogens used in the study

\begin{tabular}{llcc}
\hline Species, isolate & Origin & Inoculum density $^{\mathbf{a}}$ & Reference $^{\mathbf{b}}$ \\
\hline Acremonium cucurbitacearum & & & \\
SP-934941 & Spain & 10,000 & 4,5 \\
SP-934943 & Spain & 10,000 & 4,5 \\
Monosporascus cannonballus & & & \\
TX-921999 & Texas & 20 & 4 \\
TX-912035 & Texas & 20 & 4 \\
Rhizopycnis vagum & & & 15 \\
TX-951120 & Texas & 1,600 & 15 \\
TX-951113 & Texas & 1,600 & \\
\hline
\end{tabular}

${ }^{a}$ Inoculum density was measured in CFU per gram of soil.

${ }^{b}$ References for optimization of inoculum density. 
diameter). Pots were made of plastic and had drainage perforations at the bottom. The pots were transferred to the growth chamber (Conviron model E15; Controlled Environments Ltd., Winnipeg, Manitoba, Canada) and plants were thinned to one per pot following emergence. The pots were placed in a complete randomized block in the growth chamber. The temperature was maintained at temperatures of $30^{\circ} \mathrm{C}$ (day) and $25^{\circ} \mathrm{C}$ (night) with $12 \mathrm{~h}$ of light and dark per day. Each pot was fertilized weekly with $1 \%$ Peters mineral nutrient mixture (20:20:20 N:P:K, United Industries Corp., St. Louis, MO). Pots were broken down 28 days after seeding and the sand was gently washed from roots. Plants were stored up to $3 \mathrm{~h}$ at room temperature on aluminum trays with roots covered with wet paper towels prior to image scanning.

Sample preparation. Images of leaves and roots of each plant from all treatments were scanned using a digital scanner (Hewlett-Packard model 6100C) connected to a personal computer. The leaves from each plant were separated from the stem, arranged according to their order on the plant, and scanned together. Root systems of all plants were thoroughly but gently cleaned from any remains of sand or other particles associated with growth medium. Roots of each plant were spread on clear plastic film so individual roots and their structure would be clearly visible. All images of leaves and roots were scanned on
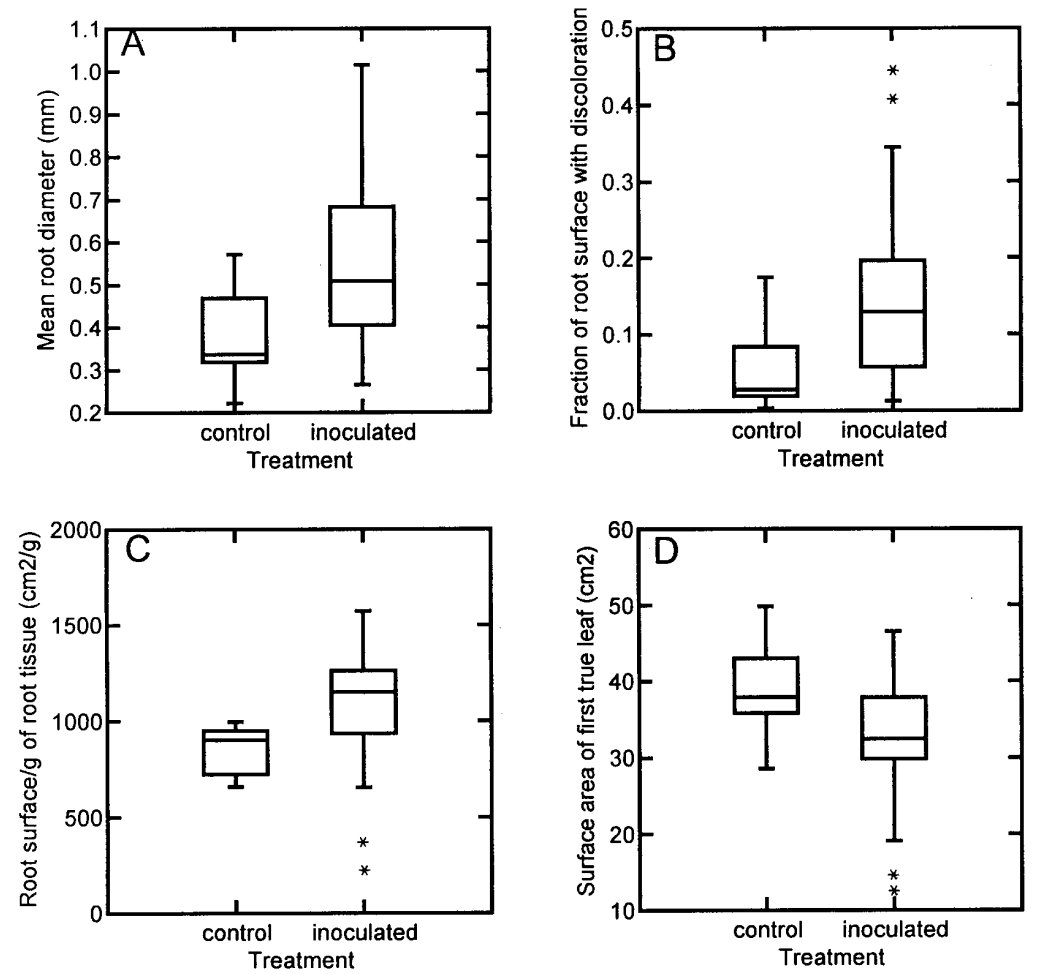

Fig. 1. Box plots showing effects of pathogen inoculation (Acremonium cucurbitacearum, Monosporascus cannonballus, and Rhizopycnis vagum) on muskmelon plant traits. Figures show median values and dispersion of $\mathbf{A}$, mean root diameter, $\mathbf{B}$, fraction of surface area of roots with discoloration, $\mathbf{C}$, root surface area to root mass ratio, and $\mathbf{D}$, surface area of first leaf in control and plants inoculated with root rot pathogens. Line in the box indicates median. Box is closed by upper and lower hinges, whiskers extend to extreme values within inner fence (inner fence is limited by points 1.5 times the distance between hinges measured in either direction from median), stars represent values between inner and outer fence (outer fence is limited by points 3 times the distance between hinges measured in either direction from median). Results represent pooled data from two experiments.

Table 2. Summary of univariate analysis of variance tests of the effects of Acremonium cucurbitacearum, Monosporascus cannonballus, and Rhizopycnis vagum on Cucumis melo plant traits evaluating effects of inoculation with root rot pathogens, pathogen species, and pathogen isolates

\begin{tabular}{|c|c|c|c|c|c|c|c|c|c|}
\hline \multirow[b]{2}{*}{ Plant traits } & \multicolumn{3}{|c|}{ Effects of inoculation $^{a}$} & \multicolumn{3}{|c|}{ Effects of species ${ }^{b}$} & \multicolumn{3}{|c|}{ Effects of isolates ${ }^{c}$} \\
\hline & $\boldsymbol{F}$ & $\boldsymbol{P}$ & $\operatorname{Rank}^{\mathbf{d}}$ & $\boldsymbol{F}$ & $\boldsymbol{P}$ & Rank & $\boldsymbol{F}$ & $\boldsymbol{P}$ & Rank \\
\hline Plant height & 0.493 & 0.4864 & $\ldots$ & 1.042 & 0.3652 & $\ldots$ & 1.170 & 0.3526 & $\ldots$ \\
\hline Plant mass & 2.965 & 0.0921 & $\ldots$ & 3.414 & 0.0462 & 5 & 4.980 & 0.0029 & 10 \\
\hline Root mass & 5.866 & 0.0211 & 8 & 1.261 & 0.2978 & $\ldots$ & 5.200 & 0.0023 & 9 \\
\hline Aboveground plant mass & 1.870 & 0.1784 & $\ldots$ & 3.692 & 0.0369 & 4 & 4.121 & 0.0076 & 13 \\
\hline Above-to-belowground plant mass ratio & 2.733 & 0.1054 & $\ldots$ & 2.299 & 0.1178 & $\ldots$ & 1.899 & 0.1319 & $\ldots$ \\
\hline Surface area of first leaf & 8.272 & 0.0062 & 4 & 2.769 & 0.0788 & $\ldots$ & 6.318 & 0.0007 & 7 \\
\hline Surface area of second leaf & 6.032 & 0.0181 & 7 & 0.712 & 0.4986 & $\ldots$ & 1.547 & 0.2130 & $\ldots$ \\
\hline Surface area of third leaf & 3.173 & 0.0818 & $\ldots$ & 2.178 & 0.1309 & $\ldots$ & 4.159 & 0.0073 & 12 \\
\hline Cumulative surface area of three true leaves & 6.398 & 0.0151 & 5 & 2.174 & 0.1313 & $\ldots$ & 4.864 & 0.0033 & 11 \\
\hline Mean surface area of a leaf & 6.398 & 0.0151 & 5 & 2.174 & 0.1313 & $\ldots$ & 4.864 & 0.0033 & 11 \\
\hline Fraction of control leaf surface area & 6.398 & 0.0151 & 5 & 3.164 & 0.0566 & $\ldots$ & 4.864 & 0.0033 & 11 \\
\hline Root surface area (RSA) & 0.001 & 0.9706 & $\ldots$ & 3.164 & 0.0564 & $\ldots$ & 3.049 & 0.0286 & 15 \\
\hline Fraction of control RSA & 6.031 & 0.0179 & 6 & 2.174 & 0.1313 & $\ldots$ & 3.049 & 0.0286 & 15 \\
\hline Fraction of RSA with discoloration & 8.816 & 0.0048 & 2 & 2.118 & 0.1379 & $\ldots$ & 12.247 & 0.0000 & 2 \\
\hline Root surface area in top $10 \mathrm{~cm}$ of soil & 1.674 & 0.2024 & $\ldots$ & 1.235 & 0.3053 & $\ldots$ & 2.199 & 0.0878 & $\ldots$ \\
\hline Fraction of plant RSA in top $10 \mathrm{~cm}$ of soil & 1.490 & 0.2288 & $\ldots$ & 5.880 & 0.0070 & 1 & 3.368 & 0.0191 & 14 \\
\hline Root surface area in top $4 \mathrm{~cm}$ of soil & 2.583 & 0.1152 & $\ldots$ & 0.950 & 0.3982 & $\ldots$ & 2.089 & 0.1020 & $\ldots$ \\
\hline Fraction of plant RSA in top $4 \mathrm{~cm}$ of soil & 0.814 & 0.3719 & $\ldots$ & 4.689 & 0.0169 & 2 & 2.785 & 0.0403 & 16 \\
\hline Root surface area-to-root mass ratio & 8.633 & 0.0052 & 3 & 3.777 & 0.0344 & 3 & 2.323 & 0.0743 & $\ldots$ \\
\hline Rhizosphere volume & 4.302 & 0.0440 & 11 & 1.677 & 0.2040 & $\ldots$ & 9.342 & 0.0001 & 6 \\
\hline Mean root diameter & 10.781 & 0.0020 & 1 & 1.679 & 0.2036 & $\ldots$ & 22.402 & 0.0000 & 1 \\
\hline Number of root tips & 4.359 & 0.0426 & 10 & 1.340 & 0.2771 & $\ldots$ & 9.694 & 0.0000 & 3 \\
\hline Total root length & 4.302 & 0.0450 & 12 & 1.677 & 0.2040 & $\ldots$ & 9.412 & 0.0001 & 5 \\
\hline Length of roots with diameter $<0.5 \mathrm{~mm}$ & 5.370 & 0.0252 & 9 & 2.392 & 0.1087 & $\ldots$ & 9.423 & 0.0001 & 4 \\
\hline Length of roots with diameter $0.5-1.0 \mathrm{~mm}$ & 0.330 & 0.5687 & $\ldots$ & 0.729 & 0.4907 & $\ldots$ & 5.629 & 0.0014 & 8 \\
\hline Length of roots with diameter $>1.0 \mathrm{~mm}$ & 2.108 & 0.1536 & $\ldots$ & 2.376 & 0.1102 & $\ldots$ & 2.243 & 0.0828 & $\ldots$ \\
\hline
\end{tabular}

${ }^{a}$ Degrees of freedom were 1, 44 for pathogen inoculation effects analyses.

${ }^{\mathrm{b}}$ Degrees of freedom were 2, 30 for pathogen species effects analyses.

${ }^{\mathrm{c}}$ Degrees of freedom were 5, 24 for pathogen isolate effects analyses.

${ }^{\mathrm{d}}$ Dependent variables were ranked with decreasing significance up to $P=0.05$. Results represent pooled data from two experiments. 
standard letter-page area $(21.6$ by $27.9 \mathrm{~cm})$ at optical resolution of 300 dots per inch (dpi) with pixel size of $0.085 \mathrm{~mm}$ and saved as digital files, which identified plant origin according to treatment and replicate.

The surface area of each leaf was measured from images using Sigma Scan Pro software (ver. 5; SPSS, Inc., Chicago). Software was calibrated in metric units according to software publisher instructions from the image of a ruler scanned at the same resolution as images of leaves and roots. The fraction of root surface area with discoloration was measured from root images following color calibration reflecting a shift in color of infected plants.

Root surface area, total root length, mean root diameter, number of root tips, and length of roots in specific diameter classes $(<0.5 \mathrm{~mm}, 0.5$ to $1.0 \mathrm{~mm}$, and $>1.0$ $\mathrm{mm})$ were measured using root analysis software WinRhizo (ver. 3.9; Regent Instruments, Quebec City, Quebec, Canada) according to manufacturer instructions. Time requirements for analysis of plant samples were recorded to evaluate sampling efficiency.

Following image scanning, all plant parts (leaves, stems, and roots) were individually packed in paper bags and marked for identification. Bags were placed in drying ovens at $65^{\circ} \mathrm{C}$. After 3 days, dry weight of plant parts was recorded for each plant identified according to treatment and replicate.

Analyses. Data were analyzed for significant differences in plant growth using ANCOVA, MANOVA, and discriminant analysis available in Systat statistical software (ver. 9; SPSS, Inc.). Discriminant analysis calculated linear discriminant functions and estimated canonical scores for each pathogen at the species or isolate level. The analysis was applied to explore the possibility that different pathogens may have specific effects on plants, affecting simultaneously a group of plant traits rather than a single characteristic. Jackknife classification procedure was used to test the likelihood of correct assignments to each group identified by discriminant analyzes (20). Prior to analyses, root diameter and rhizosphere volumes were transformed by $\log _{10}$, and fraction of root surface with discoloration were transformed by arcsin of square root to improve the normality of distribution. Transformations were not applied to variables with coefficients of variation $<20 \%$.

\section{RESULTS}

Plant response: inoculated versus noninoculated. Analysis (MANOVA) demonstrated no difference between duplicate studies; therefore, data were pooled for subsequent analysis. Analysis of variance (ANOVA) indicated significant effects in 14 of 26 analyzed plant traits in inoculated versus control plants (Table 2).
Plant traits most responsive to effects of inoculation were mean root diameter (Fig. 1A), fraction of root surface area with discoloration (Fig. 1B), root surface to root mass ratio (Fig. 1C), and surface area of first true leaf (Fig. 1D). Inoculated plants exhibited a significant increase in mean root diameter, fraction of root surface area with discoloration, root surface area to root mass ratio, and decreased leaf area of the first true leaf compared to control plants. Based on ANOVA, changes in mean root diameter of plants ranked first, as most likely to reflect effects of inoculation of the plant with a pathogen, followed by fraction of root surface with discoloration, and by surface area of first true leaf (Table 2). Root mass ranked eighth out of 26 traits analyzed as likely to indicate effects of inoculation of muskmelon with root rot pathogens. Range of distribution of each trait was significantly greater in inoculated plants than that of control plants (Fig. 1).
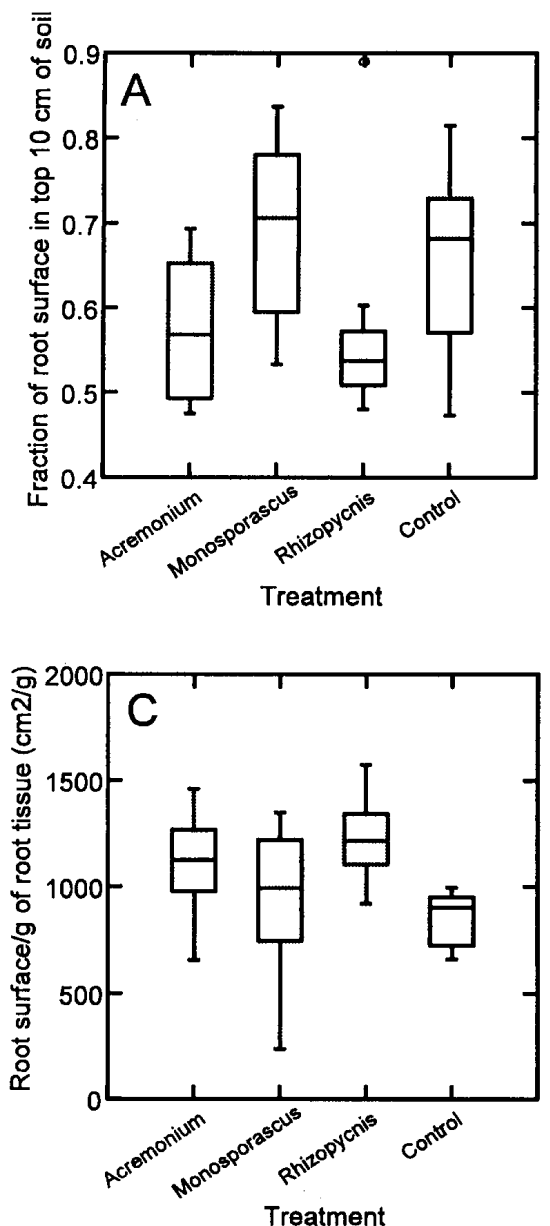

Fig. 2. Box plots showing effects of inoculation by different species of pathogens (Acremonium cucurbitacearum, Monosporascus cannonballus, and Rhizopycnis vagum) on muskmelon plant traits. Figures show median values and dispersion of $\mathbf{A}$, fraction of surface area of roots within top $10 \mathrm{~cm}$ of soil, $\mathbf{B}$, fraction of surface area of roots within top $4 \mathrm{~cm}$ of soil, $\mathbf{C}$, root surface area to root mass ratio, and $\mathbf{D}$, aboveground plant mass for control and plants inoculated with each species of root rot pathogens. Line in the box indicates median. Box is closed by upper and lower hinges, whiskers extend to extreme values within inner fence (inner fence is limited by points 1.5 times the distance between hinges measured in either direction from median), stars represent values between inner and outer fence (outer fence is limited by points 3 times the distance between hinges measured in either direction from median), and open circles represent outliers. Results represent pooled data from two experiments.

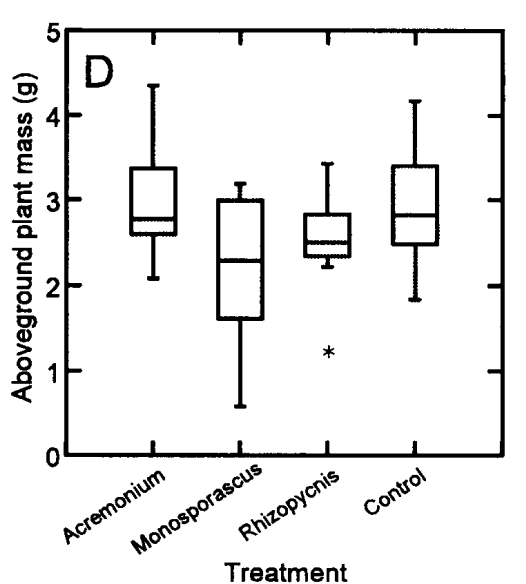

Mean root diameter of control plants ranged from 0.19 to $0.42 \mathrm{~mm}$ whereas, for to $1.25 \mathrm{~mm}$ (Fig. 1A). Fraction of surface area with discoloration ranged from 0 to $18 \%$ for control and from 10 to $80 \%$ for inoculated plants (Fig. 1B). Root $\mathrm{cm}^{2} / \mathrm{g}$ ) in control and $1,574 \mathrm{~cm}^{2} / \mathrm{g}$ (SE 47.4 $\mathrm{cm}^{2} / \mathrm{g}$ ) in inoculated plants (Fig. 1C). Mean surface area of first true leaf was significantly greater in control plants (39.3 Fig. 1D). Cumulative surface area of first three true leaves, mean leaf surface area, and fraction of control leaf surface area (to e proportional decrease in pathogen trol plants) ranked fifth as likely to reflect significant effects of inoculation (Table 2). Ranks of variables did not differ between

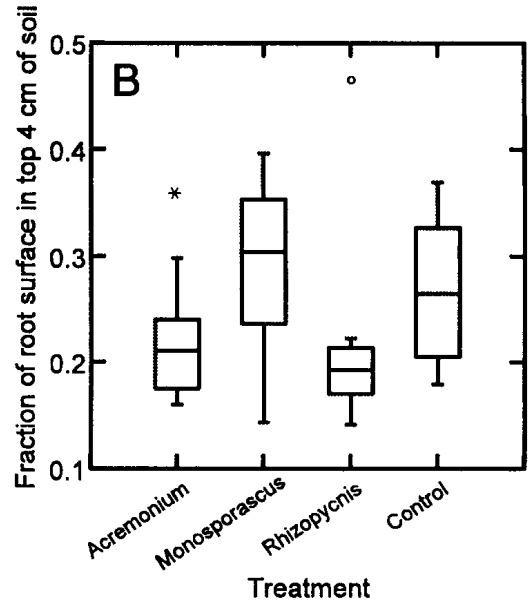

Plant Disease / January 2001 2 plants (mean $32.4 \mathrm{~cm}^{2}$ and SE $1.34 \mathrm{~cm}^{2}$; 

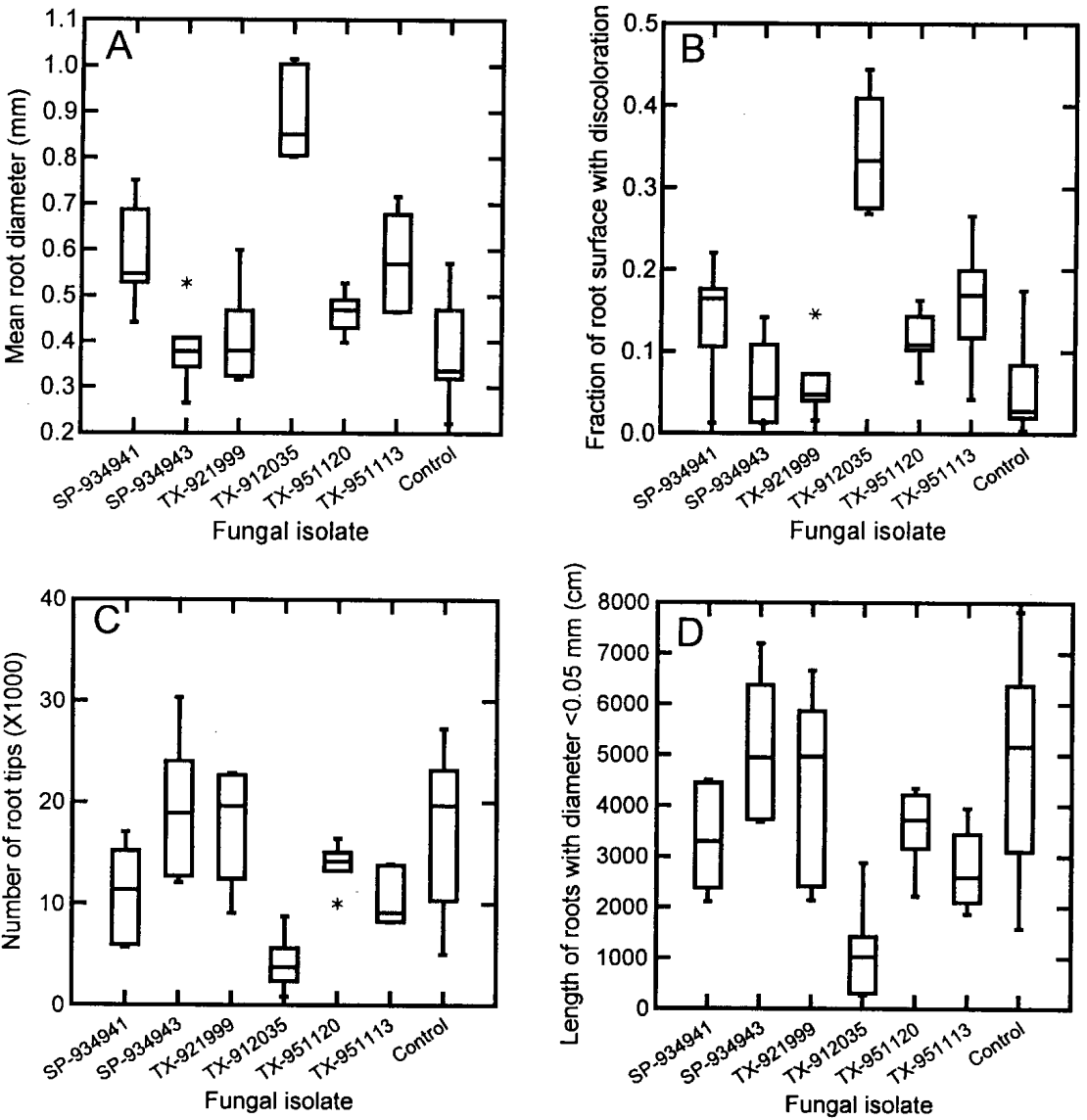

Fig. 3. Box plot showing effects of inoculation by different isolates of fungal pathogens (Acremonium cucurbitacearum, Monosporascus cannonballus, and Rhizopycnis vagum) on muskmelon plat traits. Figures show median values and dispersion of A, mean root diameter, $\mathbf{B}$, fraction of surface area of roots with discoloration, $\mathbf{C}$, number of root tips, and $\mathbf{D}$, length of roots with diameter up to $0.5 \mathrm{~mm}$ in control and plants inoculated with different isolates of root rot pathogens. Line in the box indicates median. Box is closed by upper and lower hinges, whiskers extend to extreme values within inner fence (inner fence is limited by points 1.5 times the distance between hinges measured in either direction from median), stars represent values between inner and outer fence (outer fence is limited by points 3 times the distance between hinges measured in either direction from median). Results represent pooled data from two experiments. replicate studies. Compared to control plants, inoculated plants averaged $27.6 \%$ reduction in number of root tips, $25.7 \%$ reduction in total root length, and a 30.9\% reduction in length of roots with diameters $<0.5 \mathrm{~mm}$. Although no seedling death occurred during the course of the study, random isolations from representative plants yielded colonies of the inoculated fungus.

Plant response: species of pathogen. Only 5 of 26 analyzed traits in muskmelon differed significantly due to inoculation with different species of root rot pathogen (Table 2). Significant differences among effects of pathogens were found in fraction of root surface area in top $10 \mathrm{~cm}$ of soil (Fig. 2A), fraction of root surface area in top $4 \mathrm{~cm}$ of soil (Fig. 2B), root surface to root mass ratio (Fig. 2C), and aboveground plant mass (Fig. 2D). Effects of M. cannonballus were significantly different from effects of $A$. cucurbitacearum and $R$. vagum for each of the five traits. Although $A$. cucurbitacearum and $R$. vagum did not differ in their effects on muskmelon traits, the effects were different from control plants.

Plant response: fungal isolates. Analysis at the isolate level indicated significant effects in 19 out of 26 analyzed traits in muskmelon (Table 2). The most affected plant traits were mean root diameter (Fig $3 \mathrm{~A}$ ), fraction of root surface area with discoloration (Fig. 3B), number of root tips (Fig. 3C), and length of roots with diameter $<0.5 \mathrm{~mm}$ (Fig. 3D). M. cannonballus (isolate TX-912035) caused the greatest shift in plant traits compared with all other isolates used in the study. Overall range of dispersion among effects of different isolates was significantly greater $(P<0.001)$ than dispersion observed among different species of pathogens.

Table 3. Scores of canonical discriminant functions separating effects of Acremonium cucurbitacearum, Monosporascus cannonballus, and Rhizopycnis vagum (two isolates per species) on Cucumis melo growth traits

\begin{tabular}{|c|c|c|c|c|c|c|c|c|}
\hline \multirow[b]{2}{*}{ Plant traits } & \multicolumn{4}{|c|}{ Effects of pathogen species ${ }^{a}$} & \multicolumn{4}{|c|}{ Effects of pathogen isolates $^{a}$} \\
\hline & Score 1 & Score 2 & Score 3 & $\operatorname{Rank}^{\mathbf{b}}$ & Score 1 & Score 2 & Score 3 & Rank \\
\hline Total variance accounted for by score & $70.3 \%$ & $19.9 \%$ & $9.8 \%$ & & $42.5 \%$ & $32.8 \%$ & $12.2 \%$ & $\ldots$ \\
\hline Plant height & -0.392 & 0.097 & 0.509 & 13 & -0.094 & -0.485 & -0.422 & 12 \\
\hline Root mass & 2.562 & 1.237 & -0.094 & 9 & 0.454 & 2.733 & -0.082 & 7 \\
\hline Aboveground plant mass & -1.146 & 0.053 & -0.904 & 3 & -0.165 & -1.353 & -0.256 & 3 \\
\hline Surface area of first leaf & -0.526 & 0.262 & -0.425 & 14 & -0.461 & -0.271 & -0.355 & 15 \\
\hline Surface area of second leaf & -0.598 & -0.197 & 0.063 & 15 & -0.163 & -0.701 & -0.023 & 16 \\
\hline Fraction of control leaf surface area & 1.514 & 0.265 & 0.408 & 6 & 0.430 & 1.522 & 0.173 & 10 \\
\hline Root surface area (RSA) & 4.032 & -1.152 & 4.045 & 12 & 0.769 & 4.801 & 3.989 & 13 \\
\hline Fraction of root area with discoloration & -0.254 & 0.371 & 0.470 & 17 & -0.211 & 0.066 & 0.534 & 15 \\
\hline Root surface area within top $10 \mathrm{~cm}$ of soil & -9.029 & 1.309 & -5.565 & 5 & -0.024 & -11.970 & -7.432 & 6 \\
\hline Fraction of plant RSA in top $10 \mathrm{~cm}$ of soil & 6.172 & -2.087 & 4.722 & 8 & -0.119 & 9.250 & 7.562 & 8 \\
\hline Root surface area in top $4 \mathrm{~cm}$ of soil & 5.006 & -1.882 & 3.493 & 11 & -0.903 & 7.574 & 5.752 & 7 \\
\hline Fraction of plant RSA in top $4 \mathrm{~cm}$ of soil & -6.300 & 2.363 & -3.947 & 10 & 0.914 & -10.082 & -8.172 & 4 \\
\hline Root surface area-to-root mass ratio & 2.663 & 1.459 & -0.174 & 1 & 0.489 & 3.055 & -0.540 & 1 \\
\hline Volume of rhizosphere & -0.425 & -1.297 & 5.175 & 16 & 1.128 & -2.475 & -2.281 & 14 \\
\hline Mean root diameter & 0.571 & -2.130 & -1.192 & 8 & 1.393 & -0.672 & 0.002 & 9 \\
\hline Number of root tips & 2.858 & -1.442 & -0.751 & 7 & 0.302 & 3.520 & 3.379 & 2 \\
\hline Length of roots with diameter $<0.5 \mathrm{~mm}$ & -3.944 & 1.912 & -4.090 & 4 & -1.606 & -2.582 & -0.853 & 11 \\
\hline Length of roots with diameter 0.5 to $1.0 \mathrm{~mm}$ & 1.391 & -1.086 & -0.920 & 2 & 0.610 & 1.194 & 0.590 & 5 \\
\hline
\end{tabular}

${ }^{a}$ Contribution of variables to each canonical score was standardized by within variance.

${ }^{\mathrm{b}}$ Forward stepwise method was used to identify rank of likelihood of each variable (in decreasing order) to discriminate among effects of pathogen species or isolates. Results represent pooled data from two experiments. 
Discriminant functions. Discriminant analysis of effects of different species of pathogen identified three canonical discriminant function scores that included 18 muskmelon plant traits (Table 3). Function 1 (score 1) allowed for separation of control plants from the group of inoculated plants (Fig. 4A). Functions 2 and 3 (scores 2 and 3) discriminated among effects on plants caused by pathogens at the species level (Fig. 4A). Variables that contributed most to separation of effects caused by different fungal species were root surface area to root mass ratio, length of roots with diameter from 0.5 to $1.0 \mathrm{~mm}$, aboveground plant mass, and length of root with diameter $<0.5 \mathrm{~mm}$. Jackknifed classification, based on discriminant functions, correctly associated each pathogen with its effects on inoculated plants from 79 to $100 \%$ of the time. Score 1 accounted for $70.3 \%$ of the total dispersion, score 2 for $19.9 \%$, and score 3 accounted for $9.8 \%$ of the total dispersion.

At the isolate level, three discriminant functions were identified using 18 plant traits (Table 3). Function 1 (score 1) separated control plants from inoculated plants (Fig. 4B). Functions 2 and 3 (scores 2 and 3) discriminated among effects on plant traits caused by different fungal isolates. Variables that contributed most in separating the effects of isolates were root surface area to root mass ratio, number of root tips, aboveground plant mass, and fraction of root surface area in top $4 \mathrm{~cm}$ of soil (Table 3). Jackknifed classification, based on discriminant functions, correctly associated effects of fungal isolates on plant traits from 47 to $89 \%$ of time. Score 1 accounted for $42.5 \%$ of the total dispersion, score 2 for $32.8 \%$, and score 3 accounted for $12.2 \%$ of the total dispersion. Discriminant functions classified isolates TX-912035 and TX-921999 of M. cannonballus correctly in 89 and $73 \%$ of cases, respectively. Isolates SP-934941 and SP-934943 of $A$. cucurbitacearum were classified correctly in 83 and $64 \%$ of cases. Isolates TX951113 and TX-951120 of $R$. vagum were the least likely to be classified correctly (54 and $47 \%$ cases classified correctly) compared with other isolates.

\section{DISCUSSION}

In the present study, differences between control and inoculated plants accounted for over $70 \%$ of total variance in quantitative plant traits. Although some root discoloration was observed in control plants, isolations were inclusive as to the cause. Roots of control plants are frequently occupied by Fusarium solani, which may or may not be associated with root discoloration (B. D. Bruton, unpublished data). Lower variance, up to $30 \%$, was associated with effects of different species of pathogens and analysis at the isolate level accounted for up to $45 \%$ of variance. Thus, analysis at the isolate level accounted for $50 \%$ more variance in quantitative plant traits than analyses at the species level.

The three pathogens induced significant differences in fraction of root surface area in top $10 \mathrm{~cm}$ of soil, fraction of root surface area in top $4 \mathrm{~cm}$ of soil, root surface area to root mass ratio, aboveground plant mass, and total plant mass. Proportional increase of root surface area in the top 4 and $10 \mathrm{~cm}$ of soil suggests that M. cannonballus causes significantly more damage to roots at a greater soil depth compared to $A$. cucurbitacearum and $R$. vagum. Previous studies $(4,14)$ have noted that $M$. cannonballus attacks the primary root and large secondary roots of muskmelons, support-
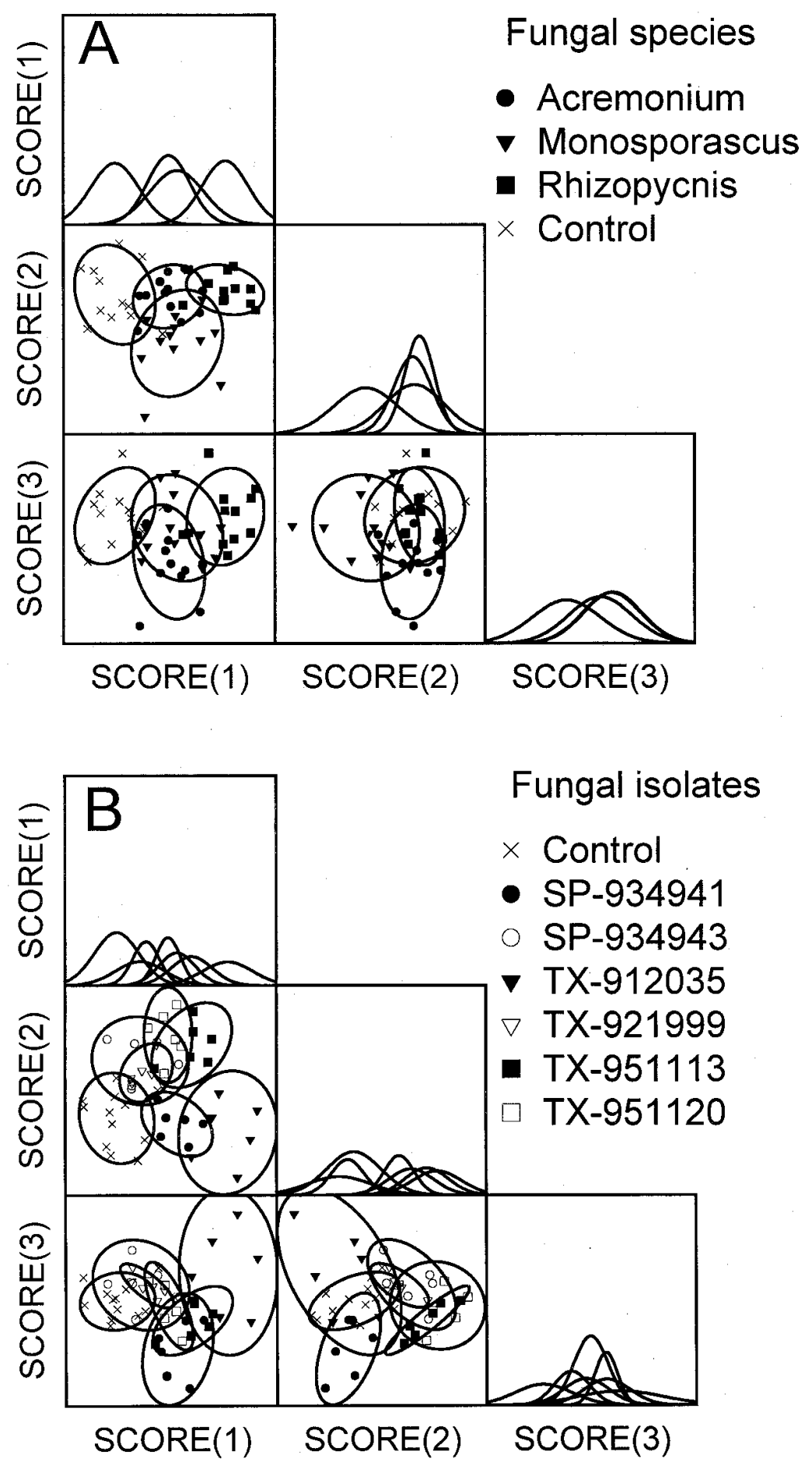

Fig. 4. Canonical scores of discriminant functions separating A, different species of root rot pathogens and control treatment and $\mathbf{B}$, different isolates of root rot pathogens and control treatment. Ellipse represents $95 \%$ Gaussian bivariate confidence for the sample. Symbols (open or filled) in B represent the same pathogen species as in A. Acremonium cucurbitacearum is represented by isolates SP-934941 and SP-934943, Monosporascus cannonballus is represented by isolates TX-912035 and TX-921999, and Rhizopycnis vagum is represented by isolates TX-951113 and TX-951120. Results represent pooled data from two experiments. 
ing conclusions in the present study. Many root rot pathogens tend to attack specific areas of the root system such as functional versus structural roots (12). Damage to the primary root appeared to cause a shift to a greater proportion of total root surface area in the top layer of soil in plants inoculated with M. cannonballus. This shift in architecture and distribution of roots in the top layer of soil may expose roots of diseased plants to greater fluctuation in moisture, temperature, and physical disturbance of soil. Plastic mulch, which maintains a more uniform soil moisture and temperature in the top soil layer, has been reported to mitigate the adverse effects of $M$. cannonballus on muskmelon plants (6). Inoculation of muskmelon with A. cucurbitacearum and $R$. vagum was associated with greater root surface to root mass ratios than in plants inoculated with $M$. cannonballus. A. cucurbitacearum and $R$. vagum caused the greatest damage to the fine roots as opposed to the tap root and main branches of the root system, which is consistent with reports on the epidemiology of $A$. cucurbitacearum $(1,4,5,9)$. These two pathogens decreased root surface area of plants to a greater extent than the decrease in root biomass.

Plant responses, analyzed at the isolate level, revealed greater differences than those observed at species level. The best measures of plant response to inoculation with fungal isolates were mean root diameter, fraction of root surface area with discoloration, number of root tips, and length of roots with diameter $<0.5 \mathrm{~mm}$. Root diameter was generally the most responsive plant trait associated with inoculation by root rot pathogens. Increased mean root diameter of inoculated plants in the present study was comparable to values reported by Crosby and Wolff (8) for different muskmelon cultivars inoculated with M. cannonballus.

Multivariate discriminant function analysis identified three discriminant functions (scores) separating species of pathogen as well as isolate effects on plant growth. Score 1 was the most useful to separate inoculated versus control plants. It was dominated by root surface area within top $10 \mathrm{~cm}$ of soil and fraction of root surface area within top $4 \mathrm{~cm}$ of soil. Scores 2 and 3 separated effects of the species as well as isolates of pathogens. Each species or isolate of pathogen affected several plant traits simultaneously with no single dominant variable. Grouping of plants based on their growth traits suggests that each pathogen isolate has distinctive multivariate effects on muskmelon. Isolates of M. cannonballus and of A. cucurbita- cearum were separated most distinctively by interaction of factors 2 and 3. Based on discriminant function scores, the two $R$. vagum isolates were more similar than isolates representing the other two genera. Using several plant traits and disease parameters, Languasco et al. (13) successfully identified races of $F$. oxysporum $\mathrm{f}$. sp. melonis with discriminant analysis. Also, discriminant function analyses have been successfully applied to taxonomic identification of biota based on various quantitative characteristics (18). In the present study, multivariate analyses of plant traits were more suitable for identifying pathogen effects on changes in plant growth compared to univariate methods.

Our study comparatively evaluated 26 plant traits for their ability to reliably reflect plant damage due to inoculation with soilborne root rot pathogens. Such comparative studies in the past have been rare and included low numbers of compared variables $(7,11,15,19)$. Development of imaging technology allows for easy collection of new variables that may be more useful in evaluation of plant disease than variables such as plant biomass, plant height, or qualitative ranking (16). Digital image analyses makes it possible to compliment qualitative disease assessment with more accurate and precise quantitative continuous scales of plant damage $(7,11,16)$. Additional studies are needed to investigate the relationship between qualitative and quantitative measures of plant damage due to soilborne pathogens. Quantitative measurement of plant traits may facilitate an objective evaluation and standardization of plant damage by soilborne root rot pathogens at the species and isolate levels (3). The present study clearly demonstrated that variation between pathogen isolates could be quantified. Consequently, this could lead to more precise quantitative estimation of virulence among isolates within a species. Standardization of plant damage would allow for interstudy comparisons and meta analyses of data. Additionally, variation in response variables could be standardized to a common scale and compared among studies, years, and location.

\section{LITERATURE CITED}

1. Alfaro, A., Armengol, J., Bruton, B. D., Gams, W., Garcia-Jiménez, J., and MartinezFerrer, G. 1996. The taxonomic position of the causal agent of Acremonium collapse. Mycologia 88:804-808.

2. Box, J. 1996. Modern methods for root investigations. Pages 193-237 in: Plant Roots. Y. Waisel, A. Eshel, and U. Kafkafi, eds. Marcel Dekker, Inc., New York.

3. Bruton, B. D. 1998. Soilborne diseases in Cucurbitaceae: pathogen virulence and host resistance. Pages 143-166 in: Cucurbitaceae '98. J. McCreight, ed. Am. Soc. Hortic. Sci Press, Alexandria, VA.

4. Bruton, B. D., Garcia-Jiménez, J., Armengol, J., and Popham, T. W. 2000. Assessment of virulence of Acremonium cucurbitacearum and Monosporascus cannonballus on $\mathrm{Cu}$ cumis melo. Plant Dis. 84:907-913.

5. Bruton, B. D., Popham, T., Garcia-Jiménez, J., Armengol, J., and Miller, M. E. 2000. Disease reaction among selected Cucurbitaceae to an Acremonium cucurbitacearum isolate from Texas. HortScience 35: 677-680.

6. Bruton, B. D., Russo, V. M., Garcia-Jiménez, J., and Miller, M. E. 1998. Carbohydrate partitioning, cultural practices, and vine decline diseases of cucurbits. Pages 189-200 in: $\mathrm{Cu}-$ curbitaceae '98. J. McCreight, ed. Am. Soc. Hortic. Sci. Press, Alexandria, VA.

7. Campbell, C. L., and Benson, M. 1994. Epidemiology and management of root diseases. Springer-Verlag, New York.

8. Crosby, K., and Wolff, D. 1998. Effects of Monosporascus cannonballus on root traits of susceptible and tolerant melon (Cucumis melo L.) cultivars. Pages 253-256 in: Cucurbitaceae '98. J. McCreight, ed. Am. Soc. Hortic. Sci. Press, Alexandria, VA.

9. García-Jiménez, J., Velázquez, T., Jorda, C. and Alfaro-García, A. 1994. Acremonium species as the causal agent of muskmelon collapse in Spain. Plant Dis. 78:416-419.

10. Gaunt, R. 1995. The relationship between plant disease severity and yield. Annu. Rev. Phytopathol. 33:119-144.

11. Hall, R. 1996. Principles and practice of managing soilborne plant pathogens. APS Press, St. Paul, MN.

12. Huisman, O. 1982. Interrelations of root growth dynamics to epidemiology of root-invading fungi. Annu. Rev. Phytopathol. 20:303-327.

13. Languasco, L., Giosue, S., Rossi, V., and Meenero, G. 1998. Statistic assignment of Fusarium oxysporum f. sp. melonis strains to races 0, 1, 2 and 1-2. J. Plant Pathol. 80:2736.

14. Mertely, J. C., Martyn, R. D., Miller, M. E., and Bruton, B. D. 1991. Role of Monosporascus cannonballus and other fungi in a root rot/vine decline of muskmelon. Plant Dis. 75:1133-1137.

15. Miller, M. E., Bruton, B. D., and Farr, D. F 1996. Rhizopycnis cucumeris, a recently described soil-borne pathogen of cucurbits. Pages 33-39 in: Melon Production Systems in South Texas. M. E. Miller, B. Bruton, L. Brandenberger, J. Batten, D. F. Farr, C Lander, G. Lester, R. Martyn, E. T. Mirkov, Y. J. Park, R. Wiedenfeld, and D. Wolff, eds. Texas Agric. Ext. Serv. 1996 Annu. Rep.

16. Nilsson, H.-E. 1995. Remote sensing and image analysis in plant pathology. Annu. Rev. Phytopahthol. 15:489-527.

17. Roberts, D., Kobayashi, D., Dery, P., and Short, N. 1999. An image analysis method for determination of spatial colonization patterns of bacteria in plant rhizosphere. Appl. Microbiol. Biotechnol. 51:653-658.

18. Sokal, R., and Rohlf, J. 1995. Biometry. Freeman and Company, New York.

19. Waisel, Y., Eshel, A., and Kafkafi, U. 1996. Plant Roots The Hidden Half. 2nd ed. Marcel Dekker, Inc., New York.

20. Zar, J. 1996. Biostatistical Analysis. PrenticeHall Canada, Inc., Toronto, Canada. 